\title{
Enfermedad hemolítica del recién nacido por anticuerpos antieritrocitarios maternos
}

Gina Laitano*

\section{RESUMEN}

La enfermedad hemolítica del recién nacido es una complicación inmunológica de la gestación, y está basada en la incompatibilidad de grupo sanguíneo entre la madre y el recién nacido. La mayoría de los antígenos que la provocan pertenecen al sistema $A B O$ y el antígeno $D$ del sistema Rh; pero en ocasiones es producida por antígenos de otros sistemas sanguíneos, como son el sistema Kell, Kidd, Duffy, Diego, Lutheran, MNS, entre otros. La madre puede inmunizarse por transfusiones previas de hematíes con antígenos no presentes en la mujer, embarazos o abortos que ocasionen hemorragia feto-materna.

Para investigar la enfermedad hemolítica del recién nacido, el médico y el laboratorio de Inmunohematología deben llevar a cabo una evaluación prenatal, que incluya aspectos obstétricos, hemoterapéuticos y estudios de laboratorio, de manera que permitan establecer el diagnóstico, orientar el pronóstico y decidir las medidas preventivas más adecuadas. Por lo que se vuelve necesaria la implementación de medidas para la prevención de la inmunización materna y así evitar la enfermedad hemolítica del recién nacido.

Palabras clave: Enfermedad hemolítica del recién nacido, hemólisis, incompatibilidad.

\section{ABSTRACT}

Hemolityc disease of the newborn is an immune complication of pregnancy; it is based in blood group incompatibility between mother and child. Most of the antigens involved in the process are part of the ABO system and, the $D$ antigen of the Rh system, in other occasions it is caused by different blood systems antigens, such as Kell, Kidd, Duffy, Diego, Lutheran and MNS, among others.The mother can be immunized by previous blood transfusions with antigens not present in her blood, pregnancy, and miscarriageswith maternal/fetal hemorrhage.

\footnotetext{
* Universidad Nacional Autónoma de Honduras. Facultad de Ciencias. Escuela de Microbiología. ginalaitano@hotmail.com
} 
In order to investigate hemolityc disease of the newborn, the physician and the Immunohematology laboratory must perform a prenatal evaluation that includes obstetrical aspects, hemo-therapy and laboratory studies to help with the diagnosis, prognosis and best decision making for preventative measures. It is necessary the implementation of measures for prevention, maternal immunization to prevent hemolytic disease of the newborn.

Key Words: Newborn haemolitycdisease, hemolysis, incompatibilty. 


\section{INTRODUCCIÓN}

La Enfermedad Hemolítica del Recién Nacido (EHRN) es una afección inmunológica aloinmune, mediada por anticuerpos maternos dirigidos contra antígenos de origen paterno presentes en los hematíes fetales y del recién nacido. Estos anticuerpos de la madre atraviesan la placenta, sensibilizan los hematíes fetales y provocan una hemólisis de estos glóbulos rojos fetales. En casos muy graves, el feto puede desarrollar hidropesía fetal y morir en el útero por falla cardiaca congestiva, secundaria a la anemia hemolítica $(3,5)$. Se han identificado varios antígenos eritrocitarios capaces de provocar una respuesta inmune y causar la EHRN, la mayoría se relaciona con los antígenos de los sistema $A B O$ y $\mathrm{Rh}$. La EHRN por incompatibilidad Rh suele ser severa, en especial por el anti D. Se puede presentar el anticuerpo anti $D$ asociado a otros anticuerpos del sistema Rh, como son el anti c y anti E. El anticuerpo anti c es capaz de provocar una EHRN severa. La EHRN por el sistema $A B O$ es la más frecuente, pero clínicamente es la menos severa. En ocasiones la EHRN es producida por antígenos de otros sistemas sanguíneos, como son el sistema Kell, Kidd, Duffy, Diego, Lutheran, MNS etc. (1, 2).

El papel del Laboratorio de Inmunohematología en la EHRN es múltiple, ya que abarca todos los aspectos relacionados con la realización de las pruebas analíticas que permiten establecer el diagnóstico, orientar el pronóstico, su prevención y tratamiento de esta complicación inmunológica de la gestación (1). La determinación seriada del título de anticuerpos maternos (Coombs inidrecto) continua siendo el primer recurso tecnológico al alcance de cualquier laboratorio para valorar el grado de aloinmunización materna y el potencial grado de afectación fetal. En esta revisión bibliográfica se identifican los factores de riesgo para que una mujer embarazada desarrolle enfermedad hemolítica perinatal, las determinaciones analíticas para el control inmunohematólogico prenatal a fin de realizar el seguimiento serológico en las gestantes $R h(D)$ negativas y en las gestantes que presentan anticuerpos anti-eritrocitarios durante el embarazo; y el control inmunohematológico postnatal para evaluar si el recién nacido necesita tratamiento.

\section{OBJETIVOS}

Describir la EHRN por incompatibilidad de grupo sanguíneo, los factores de riesgo para la inmunización y los métodos utilizados actualmente para su detección, diagnóstico y prevención. 


\section{MECANISMOSDEINMUNIZACIÓN MATERNA}

Las causas de inmunización materna son las transfusiones de hematíes con antígenos no presentes en la mujer y embarazos incompatibles 0 abortos que ocasionen hemorragia feto-materna (3).

Las gestantes deben llevar un control inmunohematológico obligatorio con el fin de la detección precoz de inmunización, identificar a las mujeres con riesgo de inducir una EHRN, y seleccionar adecuadamente a las mujeres Rh (D) negativo no sensibilizadas que pueden y deben beneficiarse de la administración profiláctica de Gammaglobulina anti-D (lgG anti-D) (1).

\section{Estímulos que favorecen la inmunización de la gestante:}

\section{a. Especificidad del anticuerpo}

La madre al exponerse a un antígeno del que carecen sus hematíes, puede desencadenar una respuesta inmune con la formación del anticuerpo correspondiente. Los anticuerpos maternos de clase lgG, dirigidos contra un antígeno bien desarrollado en los hematíes fetales pueden acabar ocasionando una EHRN. El antígeno que más frecuentemente induce una inmunización es el D. Aunque potencialmente cualquier anticuerpo puede producir esta complicación, en la práctica clínica son muy pocos los que producen una EHRN grave y, entre ellos, destacan los de especificidad anti-c, y anti-k. También la estructura, distribución tisular y la densidad del antígeno tienen un papel determinante que condiciona la producción final, o no, de la hemólisis.

\section{b. Fenómenos inmunizantes}

Es de gran importancia evitar las transfusiones de sangre Rh (D) positiva a mujeres Rh (D) negativas en edad fértil, pues el anti $D$ estimulado por transfusiones es capaz de ocasionar una EHRN muy severa. Los eritrocitos presentes concentrados en plaquetas o granulocitos, también constituyen un estímulo inmunizante para mujeres Rh (D) negativos, si los componente provienen de donantes $\mathrm{Rh}$ (D) positivos. Se recomienda que las mujeres en edad fértil sean transfundidas con eritrocitos $\mathrm{c}$ negativo y/o K negativo, si carecen de estos antígenos. En parejas que planean tener hijos, no debe transfundirse a la mujer con eritrocitos de su esposo $(1,3)$.

Por otro lado, en la mayoría de los embarazos se producen pequeñas 
hemorragias feto-maternas las cuales aumentan en frecuencia y volumen conforme avanza la gestación, alcanzando su cuota máxima en el momento del parto. Existen circunstancias y maniobras obstétricas que favorecen la intensidad de estas hemorragias, tales como la toxemia del embarazo, la extracción manual de la placenta, los abortos espontáneos y terapéuticos, el embarazo ectópico, el traumatismo abdominal, maniobra extractiva, la cesárea, la amniocentesis, la cirugía intrauterina, el feto muerto, placenta previa, entre otras $(1,3,4)$.

\section{c. Respuesta inmune}

Dependerá de varios factores, como son la inmunogenicidad del antígeno, volumen y número de episodios hemorrágicos, capacidad de respuesta de cada gestante y de que se haya realizado, o no, la profilaxis. La incompatibilidad ABO materno-fetal reduce parcialmente las posibilidades de inmunización frente a otros antígenos. El antígeno D clínicamente es el más importante, relativamente bien desarrollado en etapas precoces y puede producir hemólisis.

Los anticuerpos antieritrocitarios maternos que aparecen secundarios a un embarazo, se deben, al paso de sangre fetal hacia la circulación materna, el cual ocurre como fenómeno fisiológico de la gestación, o puede presentarse como un evento patológico. La concentración de IgG aumenta progresivamente en la primera mitad de la gestación, pero a partir de la 22-24 semanas, la concentración fetal de lgG se eleva de forma exponencial $(1,2,5)$. En la EHRN inducida por anti-K suele observarse una discordancia entre el grado de anemia encontrado y la hemólisis fetal. Igualmente, el título de anti-K puede ser paradójicamente bajo. La explicación reside en que el anti-K es capaz de inhibir la eritropoyesis fetal incidiendo en la anemia muy por encima de lo que la propia hemólisis es capaz (2).

\section{EVALUACIÓN PRENATAL}

\section{PeríodoAntenatal}

Se deben evaluar antecedentes:

1. Obstétricos: Traumatismos abdominales, abortos, estudios invasivos que puedan causar sensibilización, inmunoprofilaxis anti D, etc.

2. Hemoterapéuticos: Investigar eventos inmunizantes como las transfusiones. 
En las mujeres sensibilizadas por transfusión, puede producirse una anemia fetal más grave, ya que el estímulo antigénico primario que suponen los hematíes transfundidos es superior al que conlleva la hemorragia transplacentaria, con resultado de formación de un anticuerpo más potente capaz de inducir mayor afectación fetal (3).

\section{Estudio de Laboratorio}

Las pruebas de laboratorio a realizar en las embarazadas apoyan para:

1. Detección precoz de una inmunización materna.

2. Detección de una inmunización tardía.

\section{Estudios Serológicos:}

1. En la primera visita al obstetra (10-16 semanas de gestación) de TODAS las gestantes se debe:

- Determinar el grupoABOy Rh(D) de los padres.

- Realizar Coombs Indirecto, para investigar anticuerpos eritrocitarios (rastreo de anticuerpos irregulares) irregulares, tanto en las mujeres $\mathrm{Rh}$ (D)negativo como en las $\mathrm{Rh}(\mathrm{D})$-positivo. Se debe efectuar con células pantalla que contengan los antígenos más habitualmente implicados en la EHRN.

- Si el Coombs Indirecto resulta positivo, investigar la especificidad y título del anticuerpo.

- Una prueba importante a realizar es la prueba cruzada con muestras de sangre de la pareja. En ella se enfrentan glóbulos rojos del hombre con el suero de la mujer. De esta manera se puede evaluar incompatibilidades potenciales feto-maternas por antígenos privados y no presentes en las células pantalla $(1,3,5)$.

\section{Coombs Indirecto Negativo:}

1. Sí el rastreo de anticuerpos irregulares resulta negativo en una gestante $R h D$ positivo, realizar un manejo obstétrico preventivo. En el último trimestre (24 - 34 semanas) se debe repetir el Coombs Indirecto, pues existe la posibilidad de una inmunización durante el embarazo, especialmente si se han dado circunstancias que favorezcan una hemorragia feto - materna (traumatismo abdominal, maniobras obstetricias, etc.) o la gestante haya sido transfundida. 
2. Si el rastreo de anticuerpos irregulares resulta negativo en una gestante Rh $D$ negativo, se debe realizar mínimo una prueba de Coombs Indirecto antes de la 28 - 30 semanas de embarazo, con el objetivo de valorar la administración de IgGanti D (1,2).

\section{Coombs Indirecto Positivo:}

Si el rastreo de anticuerpos irregulares resulta positivo en una gestante Rh D negativo, es necesario llevar a cabo un programa profiláctico antenatal, con la participación del ginecólogo y el pediatra, realizando pruebas de laboratorio y exploraciones más complejas (1).

\section{Pruebas a realizar en las gestantes sensibilizadas}

- Especificidad del Anticuerpo: Evaluar si el anticuerpo identificado puede ocasionar EHRN.

- Titular el Anticuerpo y/o cuantificarlo: El título del anticuerpo en la madre, sigue siendo la técnica más sencilla al alcance de cualquier laboratorio para evaluar el grado de inmunización de la madre y el potencial grado de afectación fetal. Para una mejor correlación, las titulaciones se deben realizar en el mismo laboratorio, utilizando hematíes del mismo fenotipo y la misma tecnología, y examinando en paralelo la muestra actual con la precedente. Con el título se puede establecer el título crítico a partir del cual están indicadas otras pruebas para valorar la afectación fetal, y con el cual una vez alcanzado puede asociarse a afectación fetal (6). La primera titulación sirve de valor de referencia para las siguientes; se considera un aumento significativo del título un incremento del mismo en dos diluciones, ejemplo: un título inicial de 1/8 que se transforma en 1/32 en la siguiente titulación, lo cual puede ser indicativo de progresión de la inmunización materna y de previsible afectación fetal (6). Los laboratorios deben definir el título crítico, es decir, el título debajo del cual el feto se afecta levemente y no se justifica el uso de técnica invasivas que puedan afectar al feto a través de una hemorragia transplacentaria. Como punto de corte se toma como referencia el título de 1/128, por debajo del cual excepcionalmente se producirá afectación fetal grave (1).

- Estudio del fenotipo paterno y estimación del genotipo más probable: Es importante conocer el genotipo paterno en la pareja de una embarazada Rh(D) negativo, pues si el padre es homocigoto (DD), todos sus hijos serán Rh (D) 
positivos y tendrán el riesgo de padecer una EHRN. Si el padre es heterocigoto (Dd) habrá un 50\% de posibilidades que cada hijo sea Rh (D) positivo o negativo. Se emplea la determinación del genotipo más probable (gmp) de acuerdo a la prevalencia conocida de los haplotipos en la población. Existen técnicas con menos error como ser el PCR y el Souther - Blot(1).

- Análisis del genotipo Rh (D): Permite identificar precozmente el grupo sanguíneo fetal con técnicas moleculares, extrayendo y estudiando el ADN fetal presente en la circulación materna. Si el feto resulta $\mathrm{Rh}(\mathrm{D})$ negativo se pueden obviar otros estudios. En caso de resultar el feto Rh (D) positivo permite aplicar un protocolo profiláctico antenatal más racional, y evaluar con mayor cercanía a lo largo de la gestación para conocer la condición fetal mediante otros procedimientos diagnósticos. El análisis se puede realizar a partir de una muestra de líquido amniótico o de corion, o de plasma materno. La obtención del plasma materno evita las técnicas de obtención invasivas de las dos primeras muestras. La determinación se lleva a cabo con técnicas moleculares como el PCR, y ha sido un gran reto para la profilaxis de la EHRN y el diagnóstico prenatal. El DNA fetal comienza a detectarse a las 7 semanas de gestación, alcanzando su máximo nivel en las 8 últimas semanas $(1,2,3)$.

- Pruebas funcionales predictivas del grado de afectación fetal: Existen estudios in vitro de inmunidad celular:

- Prueba de monocapa de monocitos(MM).

- Prueba de citotoxicidad celular dependiente de anticuerpos (ADCC).

\section{Seguimiento de laboratorio en la gestante sensibilizada}

\section{Título de anticuerpos por debajo de 1/128:}

Realizar un seguimiento mensual de laboratorio y con el ginecólogo.

\section{Título de anticuerpos mayor de 1/16 y menor de 1/128:}

Realizar control serológico mensual, investigar el fenotipo/genotipo paterno y genotipo Rh (D) fetal en el segundo trimestre, además del control obstétrico.

\section{Título mayor o igual a 1/1 28:}

Llevar a cabo un control serológico mensual hasta la semana 28 , y a partir de la semana 28 hacerlo quincenal.

En las mujeres con antecedentes de EHRN se efectuará una monitorización individualizada en función de la gravedad clínica sufrida por los fetos/neonatos 
anteriores. En general se sigue el protocolo de las gestantes que alcanzan un título de $1 / 128(1)$.

Observaciones para la tipificación ABO/ Rh (D), detección e identificación de anticuerpos irregulares

Identificar correctamente las muestras de las gestantes: Incluir en las muestras los nombres y apellidos y fecha de nacimiento, y escribir en la solicitud si la gestante tiene antecedentes transfusionales y obstétricos.

Tipificación $R h(D)$ : Se recomienda emplear un reactivo monoclonal lgM que no reconozca las variantes DVI.

Sí resulta Rh (D) negativo, el equipo responsable del programa profiláctico debe informar a la gestante que es Rh (D) negativo, y por lo tanto es candidata al tratamiento con IgG anti $\mathrm{D}$ de acuerdo con el calendario previsto.

Escrutinio e identificación de anticuerpos irregulares: La técnica de elección para realizar investigar anticuerpos irregulares antieritrocitarios es la de antiglobulina indirecta (Coombs indirecto) con incubación a $37^{\circ} \mathrm{C}$.

Los hematíes (células pantalla) utilizados deben contener los siguientes antígenos: C, c, D, E, e, K, k, Fya, Fyab, Jka, Jkb, S, s, M, N y Lea. Se recomienda que los antígenos Fya, Fyb, Jka, Jkb, S y s estén presentes de forma homocigota (6).

\section{Diagnóstico postnatal de la EHRN}

Se efectúa a partir del examen clínico realizado por el médico y con los estudios inmunohematologicos llevados a cabo en el banco de sangre.

En la madre indicar la determinación del sistema $A B O$ y Rh (D), investigar en su suero la especificidad del anticuerpo eritrocitario y demostrar la presencia de anticuerpos contra el antígeno ausente.

En recién nacidos de madres $\mathrm{Rh}(\mathrm{D})$ negativo no inmunizadas, si se observan signos compatibles con EHRN o se sabe que la madre es portadora de un anticuerpo irregular capaz de producir EHRN, indicar en el neonato la determinación del sistema $\mathrm{ABO}$ y $\mathrm{Rh}(\mathrm{D})$, prueba directa de la antiglobulina en sangre del cordón, elución del anticuerpo de la muestra de cordón y fenotipo eritrocitario $(1,3)$. 
La estrategia de diagnóstico de EHRN, representa a veces una dificultad para el clínico, pues la ictericia y la hiperbilirrubinemia, muestran una gran variedad de causas, en ocasiones combinadas o distintas a la EHRN. Esto ha llevado a la práctica de efectuar de forma rutinaria la prueba directa de antiglobulina humana (Coombs directo), como tamizaje en la evaluación neonatal. Esta prueba tiene limitaciones en casos de incompatibilidad ABO, donde en más del $80 \%$ de los casos el resultado es negativo, pero si se realiza con técnica de eluido, la positividad de la prueba aumenta $(1,2,5)$.

\section{LaEHRN por Rh (D) no ha desaparecido totalmente por:}

1. Uso de dosis insuficiente de lgGanti D.

2. Omisión de la dosis preceptiva en la semana 28 de gestación.

Causas del aumento de la prevalencia de las inmunizaciones frente a otras especificidades

1. Hábito creciente de estudiar solamente a las gestantes $\mathrm{Rh}(\mathrm{D})$ negativas.

2. Costumbre de realizar el Coombs indirecto a las gestantes utilizando solamente glóbulos rojos $O$ positivos, en lugar de realizarlo con células pantalla.

3. Mayor número de transfusiones en mujeres jóvenes, sin respetar la compatibilidad Ky c.

4. Inmigración(1).

\section{Prevención de la inmunización materna:}

1. Cumplir estrictamente el programa profiláctico con lgG anti D.

2. Evitar las transfusiones en mujeres en edad reproductiva.

3. Respetar la compatibilidad cyK.

4. Educar a médicos, microbiólogos, población general y población inmigrante (1).

\section{BIBLIOGRAFÍA}

American Association of Blood Banks. Technical Manual. $12^{\text {th }}$ edition. Bethesda, Maryland, USA. 1996.

Instituto Universitario Italiano de Rosario. Curso a Distancia de Inmunohematología

y Medicina Transfusional. Argentina. 2012. Son cursos que recibo de forma virtual, el ultimo lo curse el año pasado.

Linares, Jesús. Inmunohematologia y Transfusión. Principios y Procedimientos. 1era. edición. Caracas. 1986. 
Muñiz - Díaz, Eduardo. 2007, Master en Medicina Transfusional. Universidad Autónoma de Barcelona, España.

Muñiz-Díaz E, Arilla M. Parra J et al. Protocolo de consenso del diagnóstico y prevención de la enfermedad hemolítica del recién nacido (EHRN). Revista Argentina de Transfusión 2010. Vol. XXXVI N¹.59-65.

Radillo Gonzales, Alfredo. Medicina Transfusional. 2da. edición. México. Prado. 2006. 